\title{
Photon Blockade in an Optical Cavity with One Trapped Atom
}

\author{
K. M. Birnbaum, A. Boca, R. Miller, A. D. Boozer, T. E. Northup, \\ and H. J. Kimble \\ Norman Bridge Laboratory of Physics 12-33, \\ California Institute of Technology, Pasadena, CA 91125 \\ andreea@caltech.edu
}

\begin{abstract}
We have observed photon blockade, as evidenced by the photon statistics for light transmitted by an optical cavity containing one trapped atom. The measurements also reveal the energy distribution for atomic motion in the trap.
\end{abstract}

(C)2006 Optical Society of America

OCIS codes: (020.5580) Quantum Electrodynamics; (270.5290) Photon Statistics

\section{Photon blockade in cavity QED}

The phenomenon of photon blockade, first proposed in Ref. [1] in analogy with Coulomb blockade for electrons, occurs when the absorption of a first input photon by an optical device blocks the transmission of a second one, thereby leading to nonclassical output photon statistics. One of the settings for which photon blockade has been predicted is that of a single atom in cavity quantum electrodynamics [2-4], in which the blockade is due to the anharmonicity of the Jaynes-Cummings ladder of eigenstates [5]. If an incoming photon resonantly excites the atom-cavity system from its ground state to $|1, \pm\rangle$ (where $|n,+(-)\rangle$ denotes the $n$-excitation dressed state with higher (lower) energy), then a second photon at the same frequency will be detuned from either of the next steps up the ladder, i.e. from states $|2, \pm\rangle$. In the strong coupling regime [6], for which the coherent rate of evolution $g$ exceeds the dissipative rates $\kappa$ and $\gamma$, this detuning will be much larger than the excited-state linewidths, so that the two-excitation manifold will rarely be populated. This in turn leads to the ordered flow of photons in the transmitted field, which emerge from the cavity one at a time.

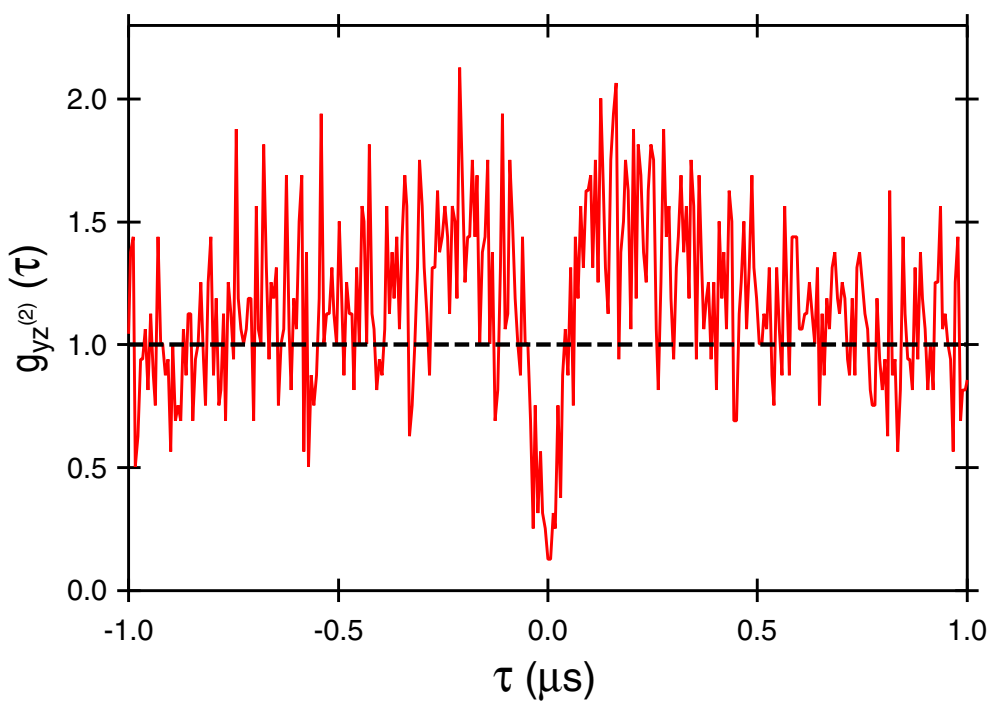

FIG. 1: Second order intensity correlation function of light emerging one photon at a time from the atom-cavity system.

We have recently observed photon blockade in the light transmitted by a high-finesse optical cavity containing one trapped Cesium atom strongly coupled to the cavity field [7]. The coherent excitation at the cavity input is near-resonant with one of the two sidebands in the previously-determined vacuum-Rabi spectrum for our system [8]. The resulting output displays both antibunching and sub-Poissonian statistics, as shown in Figure 1. The measured second order intensity correlation function demonstrates the manifestly 
nonclassical character of the output field, with $g^{(2)}(0)=(0.13 \pm 0.11)<1$ and $g^{(2)}(0)<g^{(2)}(\tau)$.

\section{Motional energy distribution}

The atom is localized within the cavity mode by the anharmonic potential of a red-detuned far-off-resonant trap. The axial and radial motion-induced modulation on the atom-cavity coupling $g$ can be observed on the cavity transmission and hence on its correlation function. Figure 2 shows a Fourier transform of the second order intensity correlation function, with a narrow peak just below the calculated maximum oscillation frequency in the axial direction, corresponding to the lowest-lying vibrational level. We use the shape of this peak to estimate that the atoms are distributed among only the lowest ten levels, with maximum energy for axial motion $E / k_{B} \sim 250 \mu \mathrm{K}$.

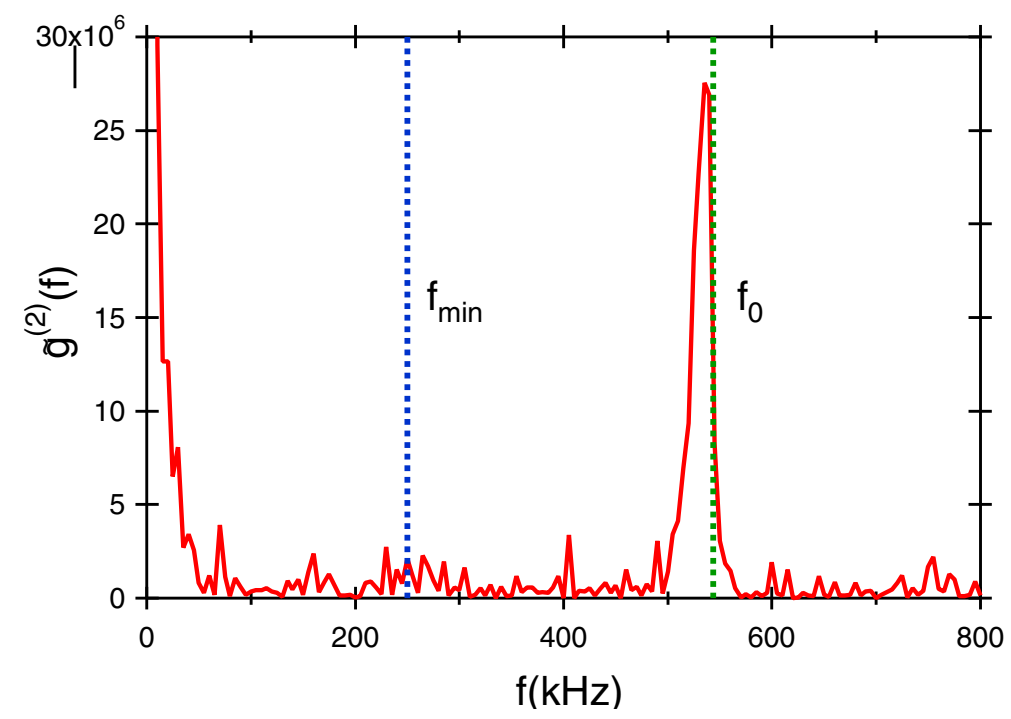

FIG. 2: Fourier transform of $g^{(2)}(\tau)$, with the dotted lines indicating the predicted maximum and minimum frequencies allowed for harmonic motion along the cavity axis.

[1] A. Imamoḡlu, H. Schmidt, G. Woods, and M. Deutsch, "Strongly Interacting Photons in a Nonlinear Cavity," Phys. Rev. Lett. 79, 1467-1470 (1997).

[2] S. Rebić, A. S. Parkins, and S. M. Tan, "Photon statistics of a single-atom intracavity system involving electromagnetically induced transparency," Phys. Rev. A 65, 063804 (2002).

[3] L. Tian and H. J. Carmichael, "Quantum trajectory simulations of two-state behavior in an optical cavity containing one atom," Phys. Rev. A 46, R6801-R6804 (1992).

[4] R. J. Brecha, P. R. Rice, and M. Xiao, " $N$ two-level atoms in a driven optical cavity: Quantum dynamics of forward photon scattering for weak incident fields," Phys. Rev. A 59, 2392-2417 (1999).

[5] E. T. Jaynes and F. W. Cummings, "Comparison of quantum and semiclassical radiation theories with application to the beam maser," Proc. IEEE 51, 89 (1963).

[6] H. J. Kimble, "Strong Interactions of Single Atoms and Photons in Cavity QED," Physica Scripta T76, 127 (1998).

[7] K. M. Birnbaum, A. Boca, R. Miller, A. D. Boozer, T. E. Northup, and H. J. Kimble, "Photon blockade in an optical cavity with one trapped atom," Nature 436, 87-90 (2005).

[8] A. Boca, R. Miller, K. M. Birnbaum, A. D. Boozer, J. McKeever, and H. J. Kimble, "Observation of the Vacuum Rabi Spectrum for One Trapped Atom," Phys. Rev. Lett. 93, 233603 (2004). 